\title{
Factor XI as a Target for New Anticoagulants
}

\author{
James C. Fredenburgh ${ }^{1,2}$ Jeffrey I. Weitz ${ }^{1,2,3}$ \\ ${ }^{1}$ Thrombosis and Atherosclerosis Research Institute, McMaster \\ University, Hamilton, Ontario, Canada \\ 2 Department of Medicine, McMaster University, Hamilton, \\ Ontario, Canada \\ ${ }^{3}$ Department of Biochemistry and Biomedical Sciences, McMaster \\ University, Hamilton, Ontario, Canada \\ Hämostaseologie 2021;41:104-110.
}

Address for correspondence Jeffrey I. Weitz, MD, 237 Barton St. E., Hamilton, Ontario, Canada L8L 2X2 (e-mail: weitzj@taari.ca).

\begin{abstract}
Keywords

- anticoagulant

- coagulation

- factor XI

- thrombosis

Despite advances in anticoagulant therapy, thrombosis remains the leading cause of morbidity and mortality worldwide. Heparin and vitamin K antagonists (VKAs), the first anticoagulants to be used successfully for the prevention and treatment of thrombosis, are associated with a risk of bleeding. These agents target multiple coagulation factors. Thus, by activating antithrombin, heparin mainly inhibits factor Xa and thrombin, whereas VKAs lower the levels of the vitamin K-dependent clotting factors. Direct oral anticoagulants, which have replaced VKAs for many indications, inhibit only factor $\mathrm{Xa}$ or thrombin. Although the direct oral anticoagulants are associated with less bleeding than VKAs, bleeding remains their major side effect. Epidemiological and animal studies have identified factor $\mathrm{XI}$ as a target for potentially safer anticoagulant drugs because factor $\mathrm{XI}$ deficiency or inhibition protects against thrombosis and is associated with little or no bleeding. Several factor XI-directed strategies are currently under investigation. This article (1) reviews the rationale for the development of factor XI inhibitors, (2) identifies the agents in most advanced stages of development, (3) describes the results of completed clinical trials and provides a summary of those underway, and (4) highlights the opportunities and challenges for this next generation of anticoagulants.
\end{abstract}

\section{Introduction}

Anticoagulants are a mainstay for the prevention and treatment of thrombosis. In the past two decades, anticoagulant strategies have evolved from a multitargeted to a targeted approach. For over half a century, heparin and vitamin $\mathrm{K}$ antagonists (VKAs) such as warfarin were the only available anticoagulants. ${ }^{1}$ VKAs target numerous steps in the coagulation cascade by attenuating the activity of the vitamin $\mathrm{K}$-dependent coagulation factors, which include factor (F) II (prothrombin), FVII, FIX, and FX. Heparin targets the active forms of these zymogens by catalyzing their inhibition by antithrombin. With advances in our understanding of the biochemistry of the coagulation system, received

January 4, 2021

accepted after revision

February 5, 2021 more specific inhibitors were developed. This started with the downsizing of heparin and the development of lowmolecular-weight heparin and fondaparinux. Findings with these smaller heparin derivatives demonstrated that anticoagulants with progressively greater selectivity for FXa were effective and safe for the prevention and treatment of thrombosis. Building on evidence that targeting specific coagulation factors was a viable approach, structure-function studies yielded small molecules that were potent and selective inhibitors of thrombin or FXa. Starting first with parenteral inhibitors of thrombin, like argatroban and bivalirudin, this culminated in the development of the direct oral anticoagulants (DOACs), including dabigatran, which inhibits thrombin, and rivaroxaban, apixaban, and

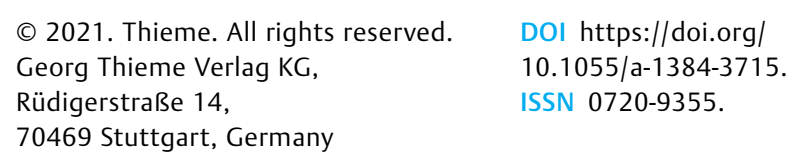

10.1055/a-1384-3715. ISSN 0720-9355. 
edoxaban, which inhibit FXa. The DOACs have now replaced VKAs for many indications. ${ }^{2}$

Better understanding of the pathogenesis of thrombosis has redirected interest to novel mechanisms that might be at play. The contact system of coagulation was ignored for many years because congenital deficiencies of FXII, high-molecular-weight kininogen (HK), and prekallikrein (PK) are not associated with bleeding and congenital deficiency of FXI is associated with only a mild bleeding diathesis. Consequently, the contact system was largely viewed as an in vitro phenomenon because physiological activators were unknown. There has been a resurgence of interest in the contact system with the recent identification of naturally occurring polyphosphates and nucleic acids as potential physiological activators of the contact system, and with increasing evidence that this system is important for thrombus stabilization and growth. Consequently, numerous animal models have established that the contact system of coagulation is more important for thrombosis than for hemostasis. Therefore, FXI has emerged as a target for development of new anticoagulants. This review identifies the unmet needs in anticoagulant therapy, summarizes the rationale for the development of FXI inhibitors, and provides evidence of initial success with FXI inhibitors in clinical trials.

\section{Unmet Needs in Anticoagulant Therapy}

Thrombosis is responsible for one in four deaths worldwide and remains a leading cause of morbidity. Although anticoagulants are a mainstay for the prevention and treatment of thrombosis, bleeding remains the major side effect. The DOACs are more convenient to administer than VKAs and are associated with less bleeding, particularly less intracranial bleeding. Nonetheless, the annual rates of major bleeding and the composite of major and clinically relevant non-major bleeding with the DOACs remain at approximately 5 and $12 \%$, respectively, in elderly patients with atrial fibrillation (AF). ${ }^{3}$ Accordingly, the fear of bleeding with the DOACs results in systemic underuse in patients with $A F$, inappropriate overuse of low-dose DOAC regimens, and reluctance to prescribe rivaroxaban on top of aspirin for secondary prevention in high-risk patients with coronary or peripheral artery disease. Therefore, there remains a need for safer anticoagulants.

\section{Contact System as a Target for Safer Anticoagulants}

The contact system was so named because of its requirement for exogenous polyanion "surfaces" such as glass, silica, kaolin, or dextran sulfate for expression of activity. The factors comprising the contact system include FXII, PK, and HK. FXI also is considered a part of the system because it binds HK, is activated by FXIla, and activates FIX. Therefore, FXI links the contact system with the intrinsic pathway. ${ }^{4,5}$

The contact system requires an autoactivation step for initiation. FXII autoactivates in the presence of polyanions. FXIIa then activates PK bound to HK, generating kallikrein (Ka). Ka reciprocally activates FXII, thereby amplifying FXIIa generation. FXIIa activates FXI, which then activates the intrinsic pathway. Back activation of FXI by thrombin connects the distal coagulation pathway with upstream events and provides the impetus to revise the waterfall or cascade models of clotting first proposed in the $1960 \mathrm{~s},{ }^{6}$ and to evaluate the role of the contact system in thrombosis.

Studies performed over the past 15 years revealed that naturally occurring polyanions serve as potent activators of the contact system. These polyanions include DNA and RNA released from dying or activated cells, neutrophil extracellular traps (NETs) extruded from activated neutrophils, ${ }^{7}$ polyphosphates released from the dense granules of activated platelets or from microorganisms, ${ }^{8}$ and misfolded proteins such as amyloid $\beta$-peptide, which is a hallmark of Alzheimer's disease. ${ }^{9}$ Because these activators are generated at the sites of cell or platelet activation, inflammation, or infection, they provide a link between coagulation and host defense mechanisms. ${ }^{10}$

Further interest in the contact system comes from epidemiological studies demonstrating a correlation between FXI levels and the risk of thrombosis. Thus, patients with FXI deficiency are at lower risk for VTE and ischemic stroke than those with normal FXI levels, but not at increased risk of serious bleeding. ${ }^{11,12}$ In contrast, patients with elevated FXI levels are at higher risk of thrombosis. ${ }^{13,14}$ Furthermore, patients with congenital FXI deficiency, which is termed "hemophilia C," have a mild bleeding diathesis and spontaneous bleeding is rare. ${ }^{15}$ Therefore, there is epidemiologic evidence that the contact system is involved in thrombosis and may be less important for hemostasis.

Studies in animals have been central to evaluating the role of the contact system in thrombosis and hemostasis. Mice deficient in FXII or FXI have normal bleeding after tail tip amputation, but exhibit attenuated clot formation at the sites of arterial or venous injury. ${ }^{4}$ Similarly, antibodies directed against FXII or FXI abrogate thrombosis in these models. ${ }^{5}$ In nonhuman primate models, antibodies directed against FXI reduce fibrin and platelet deposition on vascular grafts, whereas those directed against FXII are less effective. ${ }^{4}$ Likewise, knockdown of FXI levels with an antisense oligonucleotide (ASO) reduces arteriovenous shunt thrombosis in baboons and knockdown of FXII or FXI attenuates catheter thrombosis in rabbits. ${ }^{16,17}$ These results prompted a search for inhibitors of FXII or FXI.

\section{Separating Hemostasis from Thrombosis}

FXI inhibitors have the potential to attenuate thrombosis with little or no disruption of hemostasis. Although hemostasis and thrombosis both rely on thrombin generation and fibrin formation, the pathways differ ( - Fig. 1). Hemostasis is triggered when the hemostatic envelope of tissue factor in the adventitia of blood vessels is breached. The high concentrations of tissue factor initiate explosive thrombin generation, which results in the formation of a hemostatic plug that seals the leak. Thus, FXII is dispensable and feedback activation of FXI by thrombin is of nominal importance in hemostasis.

In contrast to hemostasis, thrombosis is usually initiated by low concentrations of tissue factor exposed at the sites of atherosclerotic plaque disruption or expressed on activated 


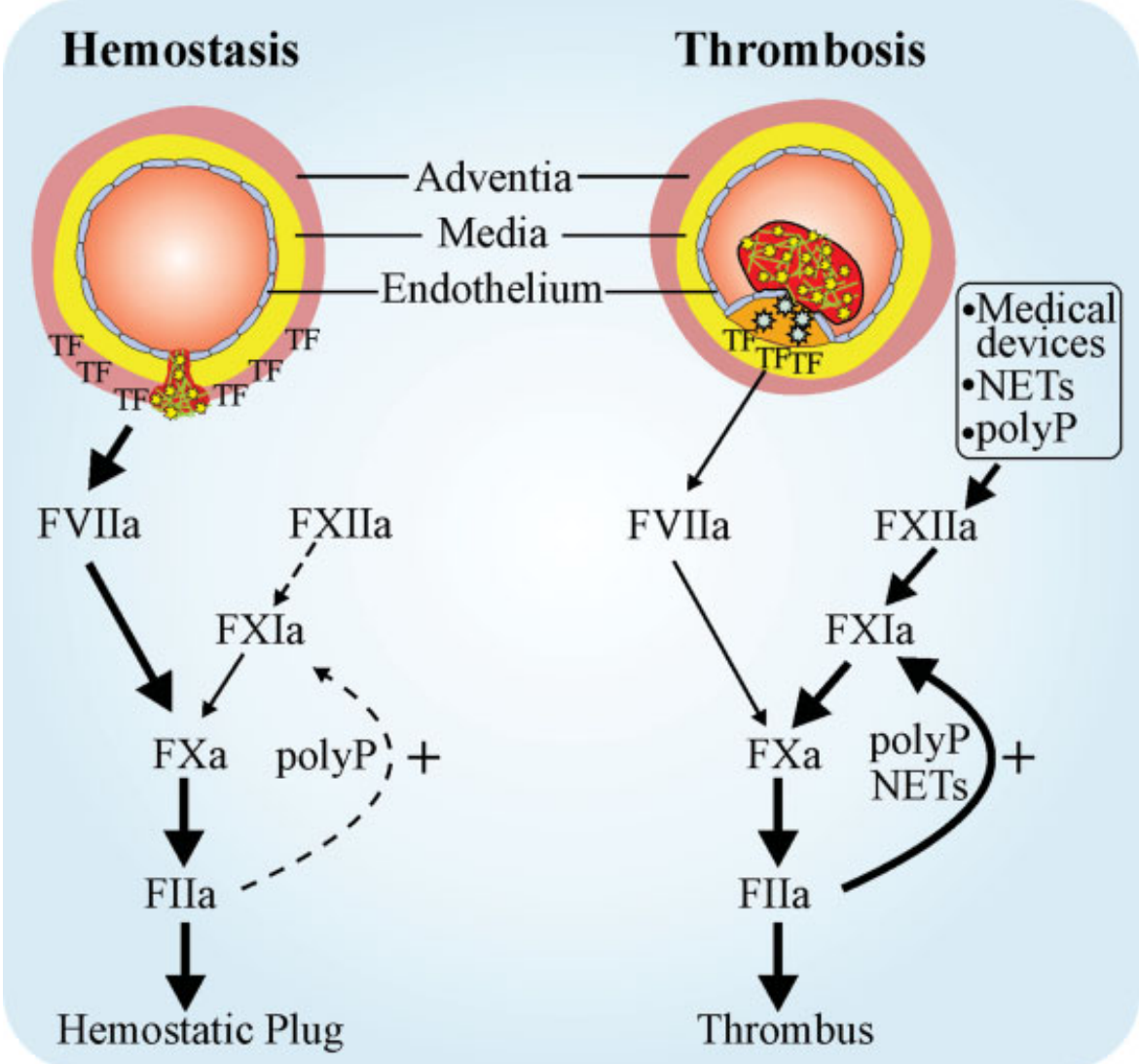

Fig. 1 Separating hemostasis from thrombosis. Blood vessels are surrounded by a hemostatic envelope of tissue factor (TF) in the adventitia. Hemostasis is triggered when the adventitia is breached by vessel injury. The high concentrations of TF surrounding the vessel initiate explosive thrombin generation via the extrinsic pathway leading to the formation of a hemostatic plug that seals the leak. FXII is dispensable for hemostasis and feedback activation of FXI by thrombin is of minor importance for amplification of thrombin generation. Thrombosis is initiated by lower concentrations of TF exposed at the site of atherosclerotic plaque rupture or on activated monocytes. The initial small amounts of thrombin feed back to activate FXI when stimulated by polyphosphates (polyP) or neutrophil extracellular traps (NETs). These polyanions and blood contacting medical devices can also bind to FXII and induce its autoactivation. FXIla then activates FXI to trigger the generation of thrombin via the intrinsic pathway.

monocytes or microvesicles that are tethered to endothelial cells. Thrombus growth and stabilization under these conditions depends on feedback activation of FXI by thrombin, which amplifies thrombin generation and fibrin formation ( - Fig. 1). By promoting the back activation of FXI by thrombin, naturally occurring polyphosphates enhance this process.

Thrombosis can also be triggered by NETs and medical devices, such as catheters or heart valves, which bind to FXII and promote its autoactivation. FXIla propagates coagulation by activating FXI, which results in thrombin generation. Back activation of FXI by thrombin amplifies this process and leads to thrombus growth and stabilization. Therefore, feedback activation of FXI is critical for thrombosis and much less important for hemostasis.

\section{Inhibitors of FXI}

With the evidence that it is more involved in thrombosis than in hemostasis, the contact system has emerged as a target for the development of new anticoagulants. ${ }^{4}$ Although FXII may be a safer target than FXI because of the lack of a bleeding diathesis associated with congenital deficiency, the epide- miological data linking coagulation factor levels with the risk of thrombosis are stronger for FXI than for FXII. ${ }^{18}$ Nonetheless, FXII inhibitors may be useful for the prevention of clotting on blood contacting medical devices or extracorporeal circuits.

Novel agents directed against FXI include inhibitors of biosynthesis, antibodies, small molecules, and derivatives of naturally occurring inhibitors. ${ }^{4}$ Differences in mode of action and pharmacological properties including oral bioavailability endow the various FXI inhibitors with divergent advantages and disadvantages. ASOs that knock down FXI levels, inhibitory antibodies, and derivatives of natural inhibitors require parenteral administration, while small molecule inhibitors can be given orally (-Table 1). Although ASOs, which are given subcutaneously, take 3 to 4 weeks to lower FXI levels into the therapeutic range, inhibitory antibodies have a rapid onset of action, particularly if given intravenously rather than subcutaneously. ASOs and inhibitory antibodies have long half-lives that enable once monthly dosing once the target level of inhibition has been achieved. Finally, FXI ASOs or inhibitory antibodies are ideal for patients at high risk for bleeding such as those with endstage renal disease (ESRD) because they are not renally 
Table 1 Properties of anticoagulants targeting FXI

\begin{tabular}{|c|c|c|c|c|c|}
\hline & Antibodies & Small molecules & Natural inhibitors & ASOs & Aptamers \\
\hline Mechanism & Bind target protein & Bind target protein & Bind target protein & Block biosynthesis & Bind target protein \\
\hline $\begin{array}{l}\text { Administration } \\
\text { route }\end{array}$ & IV or SC & IV or oral & IV & SC & IV or SC \\
\hline $\begin{array}{l}\text { Administration } \\
\text { frequency }\end{array}$ & Monthly & Daily & Daily & Weekly to monthly & Daily \\
\hline Onset of action & $\begin{array}{l}\text { Rapid } \\
\text { (hours to days) }\end{array}$ & $\begin{array}{l}\text { Rapid } \\
\text { (minutes to hours) }\end{array}$ & Rapid (minutes) & Slow (weeks) & $\begin{array}{l}\text { Rapid } \\
\text { (minutes to hours) }\end{array}$ \\
\hline Offset of action & Slow (weeks) & $\begin{array}{l}\text { Rapid } \\
\text { (minutes to hours) }\end{array}$ & Rapid (hours) & Slow (weeks) & $\begin{array}{l}\text { Rapid } \\
\text { (minutes to hours) }\end{array}$ \\
\hline Renal excretion & No & Yes & Uncertain & No & No \\
\hline CYP metabolism & No & Yes & No & No & No \\
\hline $\begin{array}{l}\text { Potential for } \\
\text { drug-drug } \\
\text { interactions }\end{array}$ & No & Yes & Unknown & No & No \\
\hline
\end{tabular}

Abbreviations: ASO, antisense oligonucleotide; CYP, cytochrome P450; IV, intravenous; SC, subcutaneous.

excreted and drug-drug interactions are unlikely because they are not metabolized via the cytochrome P450 system nor are they substrates for P-glycoprotein.

Small molecule inhibitors of FXI such as JNJ-70033093 (formerly BMS-986177), BAY 2433334, and ONO-5450598 can be given orally and have a rapid onset and offset of action, which enable once or twice daily administration. With some CYP450 metabolism and renal elimination, drug-drug interactions and accumulation in patients with renal impairment are potential complications with these agents.

Two naturally occurring Kunitz-type inhibitors against FXIa have been described. Fasxiator, an extract from the venom of the banded krait snake, binds to FXIa with high affinity and prolongs the time to occlusion in a murine $\mathrm{FeCl}_{3}$-induced carotid artery thrombosis model. ${ }^{19} \mathrm{Ir}$-CPI is a dual FXIIa and FXIa inhibitor isolated from the salivary glands of the tick Ixodes ricinus. Ir-CPI attenuated arterial and venous thrombosis in murine models, ${ }^{20}$ prolonged the time to catheter occlusion in rabbits, and reduced clotting on an extracorporeal circuit in sheep to the same extent as heparin. ${ }^{21}$ Although a phase 1 study of Ir-CPI in healthy volunteers is underway (clinicaltrials.gov; NCT04653766), neither Fasxiator nor FXI-directed aptamers ${ }^{22}$ have been evaluated in humans.

\section{Potential Indications for FXI Inhibitors}

New anticoagulants targeting FXI face an uphill battle given the success of the DOACs. The challenge will be even greater, as generic versions of the DOACs result in reduced cost and increased utilization. Consequently, FXI inhibitors will need to find niches where DOACs are contraindicated or their utility has not been established. Such indications include prevention of major adverse cardiovascular events in patients with ESRD on hemodialysis, prevention of clotting on medical devices such as central venous catheters, mechanical heart valves, or ventricular assist devices or for secondary prevention in patients with noncardioembolic stroke. ${ }^{23}$ Finally, FXI inhibitors may also be useful for stroke prevention in patients with AF who are at high risk for bleeding or may provide a safer platform than DOACs when used in conjunction with single- or dual-antiplatelet therapy.

Clinical evaluation of new anticoagulants often starts in patients undergoing joint replacement surgery because such patients are at high risk of postoperative asymptomatic deep-vein thrombosis (DVT), which can be detected on routine venography. Although these asymptomatic thrombi are of questionable clinical relevance, they enable evaluation of the antithrombotic efficacy of new anticoagulants relative to that of a comparator such as enoxaparin. In addition, excess postoperative bleeding from the wound helps identify doses of new anticoagulants that may be too high. Once effective and safe doses are identified, these doses can then be evaluated in other clinical indications.

\section{Clinical Trials with FXI Inhibitors}

Agents that inhibit FXI are at various stages of development and testing in humans. None has reached phase 3 evaluation; completed and ongoing phase 2 studies with each agent will briefly be described ( $>$ Table 2 ).

Antisense oligonucleotides: The drug that has pro-

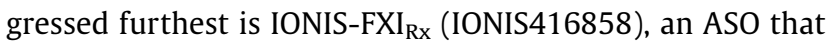
attenuates FXI biosynthesis. Maximum reductions take 3 to 4 weeks of treatment and restoration of FXI to baseline levels is delayed for several weeks after treatment is stopped. In a phase 2 study, this ASO was compared with enoxaparin for postoperative thromboprophylaxis in 300 patients undergoing elective knee arthroplasty. Patients received subcutaneous injections of IONIS-FXI $I_{R x}$ at doses of 200 or $300 \mathrm{mg}$ starting 35 days prior to surgery. ${ }^{24}$ These doses were chosen because, in a phase 1 study, they reduced FXI levels by approximately 60 and 80\%, respectively. Venography 
Table 2 Clinical trials of drugs targeting FXI

\begin{tabular}{|c|c|c|c|c|c|c|}
\hline Compound & Indication & $N$ & Comparator & Baseline therapy & Status & Registry number \\
\hline $\begin{array}{l}\text { IONIS-FXI-Rx } \\
\text { IONIS FXI-LRx } \\
\text { (BAY2976217) }\end{array}$ & $\begin{array}{l}\text { TKA } \\
\text { ESRD } \\
\text { ESRD }\end{array}$ & $\begin{array}{l}300 \\
200 \\
228\end{array}$ & $\begin{array}{l}\text { Enoxaparin } \\
\text { Placebo } \\
\text { Placebo }\end{array}$ & & $\begin{array}{l}\text { Completed } \\
\text { Completed } \\
\text { Ongoing }\end{array}$ & $\begin{array}{l}\text { NCT01713361 } \\
\text { NCT03358030 } \\
\text { NCT04534114 }\end{array}$ \\
\hline $\begin{array}{l}\text { Osocimab } \\
\text { (BAY1213790) }\end{array}$ & $\begin{array}{l}\text { TKA } \\
\text { ESRD } \\
\text { ESRD }\end{array}$ & $\begin{array}{l}813 \\
50 \\
600\end{array}$ & $\begin{array}{l}\text { Enoxaparin or } \\
\text { apixaban } \\
\text { Placebo } \\
\text { Placebo }\end{array}$ & & $\begin{array}{l}\text { Completed } \\
\text { Ongoing } \\
\text { Ongoing }\end{array}$ & $\begin{array}{l}\text { NCT03276143 } \\
\text { NCT03787368 } \\
\text { NCT04523220 }\end{array}$ \\
\hline $\begin{array}{l}\text { Abelacimab } \\
\text { (MAA868) }\end{array}$ & $\begin{array}{l}\text { TKA } \\
\text { AF }\end{array}$ & $\begin{array}{l}400 \\
48\end{array}$ & $\begin{array}{l}\text { Enoxaparin } \\
\text { Placebo }\end{array}$ & & $\begin{array}{l}\text { Ongoing } \\
\text { Ongoing }\end{array}$ & $\begin{array}{l}\text { EudraCT 2019-003756-37 } \\
\text { NCT04213807 }\end{array}$ \\
\hline AB023 (Xisomab) & $\begin{array}{l}\text { ESRD } \\
\text { PICC }\end{array}$ & $\begin{array}{l}24 \\
50\end{array}$ & $\begin{array}{l}\text { Placebo } \\
\text { None }\end{array}$ & & $\begin{array}{l}\text { Completed } \\
\text { Ongoing }\end{array}$ & $\begin{array}{l}\text { NCT03612856 } \\
\text { NCT04465760 }\end{array}$ \\
\hline $\begin{array}{l}\text { JNJ70033093 } \\
\text { (BMS-986177) }\end{array}$ & $\begin{array}{l}\text { TKA } \\
\text { Stroke }\end{array}$ & $\begin{array}{l}1,200 \\
2,350\end{array}$ & $\begin{array}{l}\text { Enoxaparin } \\
\text { Placebo }\end{array}$ & ASA \pm clopidogrel & $\begin{array}{l}\text { Ongoing } \\
\text { Ongoing }\end{array}$ & $\begin{array}{l}\text { NCT03891524 } \\
\text { NCT03766581 }\end{array}$ \\
\hline BAY 2433334 & $\begin{array}{l}\text { AF } \\
\text { Stroke } \\
\text { AMI }\end{array}$ & $\begin{array}{l}750 \\
1,800 \\
1,600\end{array}$ & $\begin{array}{l}\text { Apixaban } \\
\text { Placebo } \\
\text { Placebo }\end{array}$ & $\begin{array}{l}\text { ASA } \pm \text { clopidogrel } \\
\text { DAPT }\end{array}$ & $\begin{array}{l}\text { Ongoing } \\
\text { Ongoing } \\
\text { Ongoing }\end{array}$ & $\begin{array}{l}\text { NCT04218266 } \\
\text { NCT04304508 } \\
\text { NCT04304534 }\end{array}$ \\
\hline
\end{tabular}

Abbreviations: AF, atrial fibrillation; AMI, acute myocardial infarction; ASA, acetylsalicylic acid; DAPT, dual-antiplatelet therapy; ESRD, end-stage renal disease; PICC, percutaneously inserted central catheter; $N$, number of patients; TKA, total knee arthroplasty.

performed 8 to 12 days after surgery demonstrated comparable 30 and $27 \%$ incidences of VTE with enoxaparin and the 200-mg dose of IONIS-FXI $\mathrm{Rx}_{\text {, }}$ respectively. In contrast, the incidence of VTE was significantly reduced to $4 \%$ with the

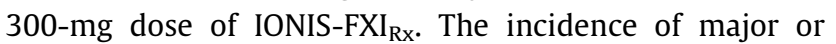
clinically relevant non-major bleeding was $3 \%$ with both doses of IONIS-FXI $\mathrm{Rx}_{\mathrm{Rx}}$ and $8 \%$ with enoxaparin, differences that were not statistically significant. The major side effect of IONIS-FXI $_{\mathrm{RX}}$ is injection-site reactions, which do not appear to be serious.

In a double-blinded, randomized, placebo-controlled pilot study in 43 patients with ESRD undergoing hemodialysis, patients were randomized to subcutaneous IONIS-FXI $\mathrm{Rx}_{\mathrm{x}}(200$ or $300 \mathrm{mg}$ twice weekly for the first week and once weekly thereafter) or placebo for 12 weeks (NCT02553889). ${ }^{25}$ At week 13 , the 200- and 300-mg doses of IONIS-FXI $\mathrm{Rx}_{\mathrm{Rx}}$ significantly $(p \leq 0.001)$ reduced mean FXI activity by 56 and $71 \%$, respectively, compared with a $4 \%$ reduction with placebo. Visual inspection of the air traps and dialysis membranes revealed less clotting with IONIS-FXI $\mathrm{Rx}_{\mathrm{Rx}}$ than with placebo even though all patients received heparin during dialysis.

Other studies in patients with ESRD on hemodialysis are

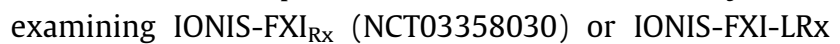
(BAY2976217), a second-generation ASO with an N-acetyl galactosamine conjugate (NCT04534114). The increased potency of the latter agent enables once monthly administration of lower doses, thereby reducing the potential for injection-site reactions.

Osocimab (BAY 1213790): A fully human IgG1 monoclonal antibody, osocimab binds to FXIa and blocks its activity. In a phase 2 noninferiority study, osocimab was compared with enoxaparin and apixaban for thromboprophylaxis in patients undergoing elective knee arthroplasty (NCT03276143). ${ }^{26}$ A total of 813 patients were randomized to single intravenous osocimab postoperative doses of 0.3 ,
$0.6,1.2$, or $1.8 \mathrm{mg} / \mathrm{kg}$; single preoperative osocimab doses of 0.3 or $1.8 \mathrm{mg} / \mathrm{kg}$; or $40 \mathrm{mg}$ of subcutaneous enoxaparin once daily or $2.5 \mathrm{mg}$ of oral apixaban twice daily until venography. Major or clinically relevant non-major bleeding was observed in up to $4.7 \%$ of those receiving osocimab, $5.9 \%$ receiving enoxaparin, and $2 \%$ receiving apixaban; all bleeding events were limited to the surgical site and there was no intracranial bleeding or bleeding into another critical site. Given postoperatively, the $0.6-, 1.2-$, and $1.8-\mathrm{mg} / \mathrm{kg}$ doses of osocimab met the criteria for noninferiority compared with enoxaparin at the prespecified noninferiority margin of $5 \%$. Although the preoperative $1.8 \mathrm{mg} / \mathrm{kg}$ dose of osocimab met the criteria for superiority compared with enoxaparin, the $4.7 \%$ rate of major of clinically relevant bleeding identified the 0.6 - and $1.2-\mathrm{mg} / \mathrm{kg}$ doses as those most promising for future investigation. A 6-month study comparing subcutaneous osocimab at these two doses with placebo in patients with ESRD undergoing hemodialysis is underway (NCT04523220). Designed primarily to evaluate safety, the primary endpoints of the study are the rates of the composite of major and clinically relevant non-major bleeding and of adverse events.

Abelacimab (MAA868): Abelacimab is a fully human IgG1 antibody that binds to FXI with high affinity and locks it in the zymogen conformation. ${ }^{27}$ Once bound to FXI, abelacimab inhibits its activation by FXIla or thrombin. Abelacimab also inhibits FXIa. Abelacimab is being compared with placebo in a pilot study in patients with AF who are at low risk of thromboembolism (NCT04213807). Patients receive once monthly subcutaneous injections of to abelacimab at three different dose levels or placebo for 3 months. The primary outcome is the extent of FXIa inhibition at trough antibody concentrations. In a phase 2 study, abelacimab is being compared with enoxaparin for postoperative thromboprophylaxis in approximately 400 patients undergoing elective 
knee arthroplasty (EudraCT number 2019-003756-37). Patients are randomized to single intravenous doses of abelacimab in one of three different doses or to subcutaneous enoxaparin. The results of this study will inform abelacimab dose selection for other indications.

AB023: A humanized version of 14E11, an antibody that binds to the apple 2 domain of FXI, AB023 inhibits FXI activation by FXIla but not by thrombin. Therefore, АB023 effectively functions as FXIIa inhibitor. ${ }^{28}$ In a small phase 2 study, 24 patients with ESRD undergoing heparin-free hemodialysis were randomized in a 2:1 manner to intravenous AB023 (at doses of 0.25 or $0.5 \mathrm{mg} / \mathrm{kg}$ ) or placebo (NCT03612856). AB023 was well tolerated and decreased dialyzer clotting and the need for dialysis circuit changes and saline flushes in a dose-dependent manner. A phase 2 study comparing $\mathrm{AB} 023$ with placebo for the prevention of central venous catheter thrombosis is underway (NCT04465760).

JNJ70033093 (BMS-986177): A potent and selective small molecule inhibitor of FXIa, JNJ70033093 binds reversibly to the active site of FXIa and inhibits its activity. ${ }^{29,30}$ Two phase 2 trials are underway. One study is comparing JNJ70033093 with enoxaparin for postoperative thromboprophylaxis in up to 1,200 patients undergoing elective knee arthroplasty (NCT03891524). Twice daily doses of JNJ70033093 ranging from 25 to $200 \mathrm{mg}$ and once daily doses of 25,50 , or $100 \mathrm{mg}$ are being evaluated. The second phase 2 study is comparing JNJ70033093 with placebo for secondary stroke prevention in up to 2,250 patients with noncardioembolic stroke or transient ischemic attack (NCT03766581). All patients receive aspirin plus clopidogrel for 21 days followed by aspirin alone thereafter. The primary endpoint is the 90-day rate of recurrent overt stroke or covert stroke detected by repeat MRI of the brain.

BAY 2433334: Like JNJ70033093, BAY 2433334 is an active site-directed, small molecule inhibitor of FXIa. ${ }^{31}$ Three phase 2 trials with BAY 2433334 are underway as part of the PACIFIC (Phase 2 Program of Anticoagulation via Inhibition of FXIa by the oral Compound BAY 2433334) program. PACIFIC-AF will compare once daily doses of BAY 2433334 (either 20 or $50 \mathrm{mg}$ ) with apixaban in 750 patients with nonvalvular AF (NCT04218266). PACIFIC-STROKE will compare BAY 2433334 at once daily doses of 10,20 , or $50 \mathrm{mg}$ with placebo on top of antiplatelet therapy in 1,800 patients with noncardioembolic stroke (NCT04304508). PACIFIC-AMI will compare BAY 2433334 at once daily doses of 10,20 , or $50 \mathrm{mg}$ with placebo on top of dual-antiplatelet therapy for the prevention of major adverse cardiac events in 1,600 patients with acute MI (NCT04304534).

\section{Conclusions and Future Directions}

In the last decade, DOACs have revolutionized anticoagulant therapy for both patients and practitioners. It appears that yet another breakthrough is imminent as factors upstream of thrombin and FXa become the target of new anticoagulants. It took just over a decade from the initial concept to advance agents targeting FXI into clinical trials to prevent thrombosis in humans. ${ }^{24,32}$ Phase 2 studies with two novel agents,
IONIS-FXI $I_{R x}$ and osocimab, provide proof-of-concept for targeting FXI, and several other agents have advanced to phase 2. If these initial successes are indicative, upstream inhibition of FXI may prove to be at least as effective as downstream inhibition at the level of FXa alone or combined inhibition of FXa and thrombin. The most likely indications will be those where the DOACs have yet to be tested such as secondary prevention in patients with noncardioembolic stroke or prevention of major adverse vascular events in patients with ESRD undergoing hemodialysis, or where the DOACs have failed such as prevention of clotting on medical devices. Head-to-head trials comparing FXI inhibitors with DOACs will require large numbers of patients to determine whether FXI inhibitors are at least as effective as DOACs but associated with less bleeding. While such trials may require large numbers of patients, there are sufficient other indications for FXI-directed agents to take hold. Consequently, the upcoming decade may witness advances in anticoagulant therapy beyond those achieved with the DOACs. Success in targeting FXI could herald an end to the more than 70-year stronghold of thrombin and FXa as targets for anticoagulants.

Note

J.I.W. holds the Canada Research Chair (Tier 1) in Thrombosis and the Heart and Stroke Foundation. J.C.F. Mustard holds Chair in Cardiovascular Research at McMaster University. This work was supported, in part, by grants from the Heart and Stroke Foundation of Canada (G-16-00013163) and the Canadian Institutes of Health Research (FDN-159928).

\section{Authors' Contributions}

J.C.F. conceived, wrote, and reviewed the manuscript.

J.I.W. conceived, wrote, and reviewed the manuscript.

\section{Conflict of Interest}

J.I.W. reports personal fees from Anthos, Bayer, Boehringer Ingelheim, Bristol-Myers Squibb, Daiichi-Sankyo, Ionis, Janssen, Novartis, Pfizer, PhaseBio, and Servier, outside the submitted work. J.C.F. reports no conflict of interest.

\section{References}

1 Hirsh J, Eikelboom JW, Chan NC. Fifty years of research on antithrombotic therapy: achievements and disappointments. Eur J Intern Med 2019;70:1-7

2 Weitz JI, Harenberg J. New developments in anticoagulants: past, present and future. Thromb Haemost 2017;117(07):1283-1288

3 Ruff CT, Giugliano RP, Braunwald E, et al. Comparison of the efficacy and safety of new oral anticoagulants with warfarin in patients with atrial fibrillation: a meta-analysis of randomised trials. Lancet 2014;383(9921):955-962

4 Tillman BF, Gruber A, McCarty OJT, Gailani D. Plasma contact factors as therapeutic targets. Blood Rev 2018;32(06):433-448

5 Grover SP, Mackman N. Intrinsic pathway of coagulation and thrombosis. Arterioscler Thromb Vasc Biol 2019;39(03): 331-338

6 Gailani D, Broze GJ Jr. Factor XI activation in a revised model of blood coagulation. Science 1991;253(5022):909-912 
7 Sorvillo N, Cherpokova D, Martinod K, Wagner DD. Extracellular DNA NET-Works with dire consequences for health. Circ Res 2019; 125(04):470-488

8 Baker CJ, Smith SA, Morrissey JH. Polyphosphate in thrombosis, hemostasis, and inflammation. Res Pract Thromb Haemost 2018; 3(01):18-25

9 Zamolodchikov D, Renné T, Strickland S. The Alzheimer's disease peptide $\beta$-amyloid promotes thrombin generation through activation of coagulation factor XII. J Thromb Haemost 2016;14(05): 995-1007

10 Spronk HMH, Padro T, Siland JE, et al. Atherothrombosis and thromboembolism: Position paper from the second Maastricht Consensus Conference on Thrombosis. Thromb Haemost 2018; 118(02):229-250

11 Preis M, Hirsch J, Kotler A, et al. Factor XI deficiency is associated with lower risk for cardiovascular and venous thromboembolism events. Blood 2017;129(09):1210-1215

12 Georgi B, Mielke J, Chaffin M, et al. Leveraging human genetics to estimate clinical risk reductions achievable by inhibiting factor XI. Stroke 2019;50(11):3004-3012

13 Yang DT, Flanders MM, Kim H, Rodgers GM. Elevated factor XI activity levels are associated with an increased odds ratio for cerebrovascular events. Am J Clin Pathol 2006;126(03):411-415

14 Meijers JC, Tekelenburg WL, Bouma BN, Bertina RM, Rosendaal FR. High levels of coagulation factor XI as a risk factor for venous thrombosis. N Engl J Med 2000;342(10):696-701

15 Duga S, Salomon O. Congenital factor XI deficiency: an update. Semin Thromb Hemost 2013;39(06):621-631

16 Zhang H, Löwenberg EC, Crosby JR, et al. Inhibition of the intrinsic coagulation pathway factor XI by antisense oligonucleotides: a novel antithrombotic strategy with lowered bleeding risk. Blood 2010;116(22):4684-4692

17 Yau JW, Liao P, Fredenburgh JC, et al. Selective depletion of factor $\mathrm{XI}$ or factor XII with antisense oligonucleotides attenuates catheter thrombosis in rabbits. Blood 2014;123(13):2102-2107

18 Weitz JI, Fredenburgh JC. 2017 Scientific Sessions Sol Sherry Distinguished Lecture in Thrombosis: Factor XI as a target for new anticoagulants. Arterioscl Thromb Vasc Biol 2018;38(02): 304-310

19 Chen W, Carvalho LP, Chan MY, Kini RM, Kang TS. Fasxiator, a novel factor XIa inhibitor from snake venom, and its site-specific mutagenesis to improve potency and selectivity. J Thromb Haemost 2015;13(02):248-261

20 Decrem Y, Rath G, Blasioli V, et al. Ir-CPI, a coagulation contact phase inhibitor from the tick Ixodes ricinus, inhibits thrombus formation without impairing hemostasis. J Exp Med 2009;206 (11):2381-2395

21 Demoulin S, Godfroid E, Hermans C. Dual inhibition of factor XIIa and factor XIa as a therapeutic approach for safe thromboprotection. J Thromb Haemost 2021;19(02):323-329

22 Chabata CV, Frederiksen JW, Sullenger BA, Gunaratne R. Emerging applications of aptamers for anticoagulation and hemostasis. Curr Opin Hematol 2018;25(05):382-388

23 Mackman N, Bergmeier W, Stouffer GA, Weitz JI. Therapeutic strategies for thrombosis: new targets and approaches. Nat Rev Drug Discov 2020;19(05):333-352

24 Büller HR, Bethune C, Bhanot S, et al; FXI-ASO TKA Investigators. Factor XI antisense oligonucleotide for prevention of venous thrombosis. N Engl J Med 2015;372(03):232-240

25 Bethune C, Walsh M, Jung B, Yu R, Geary RS, Bhanot S. Pharmacokinetics and pharmacodynamics of Ionis-FXIRX, an antisense inhibitor of factor XI, in patients with end-stage renal disease on hemodialysis. Blood 2017;130:1116

26 Weitz JI, Bauersachs R, Becker B, et al. Effect of osocimab in preventing venous thromboembolism among patients undergoing knee arthroplasty: the FOXTROT randomized clinical trial. JAMA 2020;323(02):130-139

27 Koch AW, Schiering N, Melkko S, et al. MAA868, a novel FXI antibody with a unique binding mode, shows durable effects on markers of anticoagulation in humans. Blood 2019;133(13):1507-1516

28 Lorentz CU, Verbout NG, Wallisch M, et al. Contact activation inhibitor and factor XI antibody, AB023, produces safe, dosedependent anticoagulation in a phase 1 first-in-human trial. Arterioscler Thromb Vasc Biol 2019;39(04):799-809

29 Wong P, Crain E, Dilger A, et al. Small-molecule factor XIa inhibitor, BMS-986177/JNJ-70033093, prevents and treats arterial thrombosis in rabbits at doses that preserve hemostasis. Res Pract Thromb Haemost 2020;4; Suppl. 1, Abstract PB0121

30 Perera V, Abelian G, Li D, et al. Single-dose pharmacokinetics of BMS-986177/JNJ-70033093 in participants with mild or moderate hepatic impairment compared to healthy participants. Eur Heart J 2020;41; Issue, Suppl. 2; Abstract ehaa946.3371

31 Thomas D, Kanefendt N, Schwers S, Unger S, Yassen A, Boxnick S. First evaluation of the safety, pharmacokinetics and pharmacodynamics of BAY 2433334 a Small Molecule Targeting Coagulation Factor XIa in Healthy Young Male Participants. Res Pract Thromb Haemost2020;4; Issue, Suppl. 1; Abstract PB0243

32 Gruber A, Hanson SR. Factor XI-dependence of surface- and tissue factor-initiated thrombus propagation in primates. Blood 2003; 102(03):953-955 